\title{
Creating synergies between traditional crafts and Fablab Making
}

\section{Exploring digital mold-making for glassblowing}

\author{
Nicolas Padfield \\ Fablab RUC \\ Denmark \\ nicolas@padfield.dk
}

\author{
Mads Hobye \\ Computer Science, \\ Fablab RUC \\ Denmark \\ mads@hobye.dk
}

\author{
Michael Haldrup \\ Performance- \\ design, RUC \\ Denmark \\ mhp@ruc.dk
}

\author{
Jason Knight \\ Fablab RUC \\ Bhutan \\ jasonk.mandin@ \\ gmail.com
}

\author{
Maja Fagerberg Ranten \\ Computer Science, \\ RUC \\ Denmark \\ mranten@ruc.dk
}

\begin{abstract}
Traditional crafts and the Maker movement have in the last decade to some extent been evolving in parallel, with little intermingling. We held an experimental five day workshop with six experienced craftspeople "traditional" glassblowers - while providing modern digital Fablab production apparatus; specifically 3D printers and CNC (Computer Numerical Control) routers to explore possibilities and synergies in working with a traditional craft in conjunction with new generalized digital production possibilities. This paper summarizes seven generalisable takeaways that highlight relevant reflections on the potential for cross-fertilization and learning, enriching the repertoire of both the "traditional" craft and the Fablab, based on a shared interest in exploring aesthetic material exploration and production.
\end{abstract}

\section{Author keywords}

Making; Fablabs; digital fabrication; traditional craft; craftsmanship; glass blowing; form making; CNC cutting; 3D printing; glass; disruption; synergy

This work is licensed under a Creative Commons Attribution International 4.0 License.

FabLearn Europe'18, June 18, 2018, Trondheim, Norway (C) 2018 Copyright is held by the owner/author(s). ACM ISBN 978-1-4503-5371-7/18/06. https://doi.org/10.1145/3213818.3213821

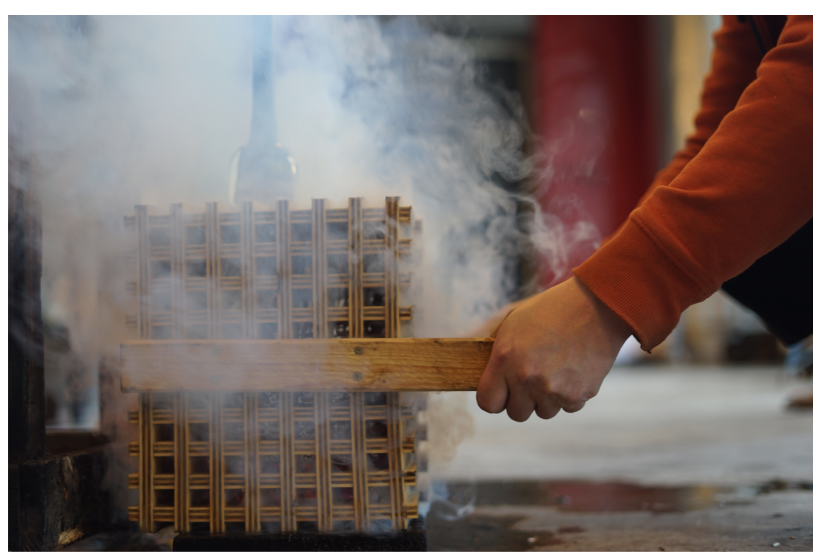

Figure 1. Moulding glass in a form made of $\mathrm{CNC}$ cut plywood, steam and smoke escaping.

\section{INTRODUCTION}

The Maker movement [11,1] has somewhat been a hobbyist movement initiated by the creative middle class, but is now gaining a stronger foothold within academia [22] as a foundation for learning through making and enabling non-technical people to prototype with technology and materials [24].

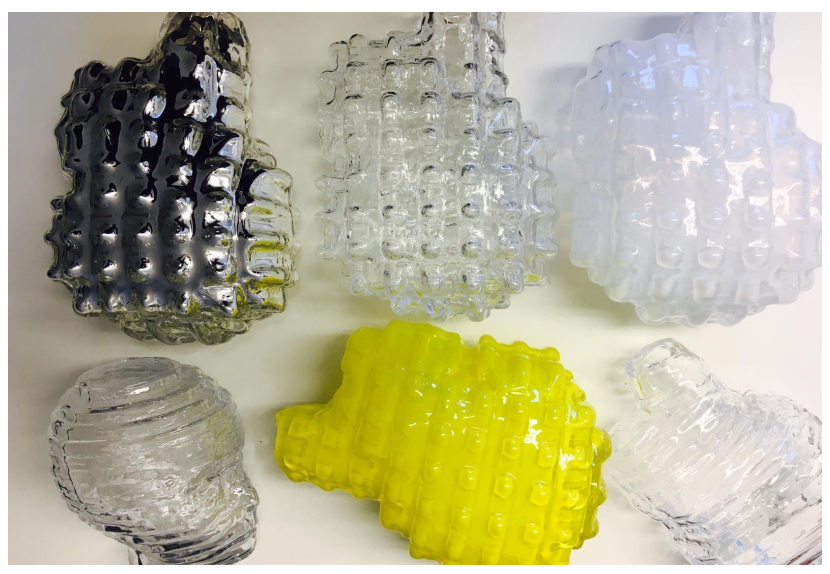

Figure 2. Resulting glass artefacts. 
For Kalil [18] makers are "people who design and make things on their own time because they find it intrinsically rewarding to make, tinker, problem-solve, discover, and share what they have learned". In that sense the "maker movement' signals a broad revolution in manufacturing partly drawing on a resurgence of DIY cultures and returns to 'crafting' [12]. Hence, the 'maker movement' has flourished through the last decade, not least because of the increased availability of working spaces such as the increasing number of Fablabs [5] around the world.

Fablabs, often growing from technologists, hacker culture and computer scientists, utilise computer controlled machines, almost to a fault. It is often easy to recognise an artefact created in a Fablab because of the specific processes available, which form both the aesthetics and the functionality. Laser cutting, 3D print, CNC milling and Arduino are common, other materials and processes less common. The movement has thus evolved in parallel with and, to some extent, without the accumulated knowledge and wider palette of processes and materials available to, "traditional" craft such as woodworking, ceramics or metalworking. In other words Fablabbing and Making have to some extent evolved in separate "silos", and while new domains and crafts are increasingly being explored by DIY makers, at the same time as new digital technologies for fabrication are being introduced, there is a possibility that they may grow even further apart. We wondered if it would be possible to challenge this tendency and find ways to build bridges and utilize potential synergies between the two worlds.

In other words we need to explore more fully how 'Making' is related to the tacit knowledge accumulated by crafting practices [15,17], and transplant this to current understandings of Making in a Fablabbing context.

\section{Methodology: Workshop as a shared learning space} Traditional learning formats sometimes have the connotations of learning as a transfer of knowledge from a skilled teacher to a student, typically within an institutional frame with clear assessment criteria [8], i.e. concepts like scaffolding [25] and nearest Zone of proximal development [26]. However as Martin [22] points out (see also Ingold on 'learning to learn' [16]), another set of qualities are at play in the Maker perspective. Play itself becomes a dominant way of experimenting while learning through Making. The "students" have a substantial say in what and how they are going to make. Learning happens in linked communities across disciplines. Learning within this workshop was thus a product of a shared playful approach to experimenting with techniques across different disciplines. Concretely, the following four principles framed the creative learning process:

- Provide space for multiple stakeholders to have a shared conversation.

- Have a wide array of machinery and tools from both fields available.

- Have skilled technical support structures around both fields as equal participants in the workshop.

- Create a framing to inspire possible outcomes, but give full autonomy to emergent collaborations between different stakeholders.

The four principles served as a basis for our research approach. Gaver [6], Redström, Zimmerman [27] argue for research through design as a way to engage with practical design explorations as a route of discovery to create knowledge contributions that are generative for others in their design processes. Methodologically this project has been framed as an open minded discovery process whereas this paper represents a set of takeaways as a simple form of annotations on the overall portfolio of experiments done during the exploration. While the case is about glassblowing, the takeaways are framed in such a way that they are generative for others [7] across a potentially wide range of craft-like disciplines and Maker endeavours.

\section{Glassblowing}

To become a master glassblower requires years of practice. The basis of glassblowing techniques have been relatively consistent for decades. There is a craftsman praxis, generations of embodied knowledge, [23] which partly already defines itself as in opposition to machine and factory produced glass. How do we bring machines and robots into this self-understanding with the framing of possibilities, not threats?

Traditional glassblowing is essentially a technique in which a glassblower picks up molten glass from a furnace with a pipe (a hollow metal rod). By blowing into the pipe while continuously turning the pipe, the glass blower is able create a ball shaped form which can be shaped either by expanding it further in forms, or by shaping it by hand with metal tools and wet paper.

To gain a greater understanding of the possibilities in a meeting of cultures, Fablab RUC coordinated a Maker/Fablab workshop for artists and researchers in 
glass blowing with and at the Glass Factory, a 'living museum' with glassblowing studio facilities.

\section{WORKSHOP: EXPLORING DIGITAL MOLD-MAKING}

The five day workshop with eight participants was held at the Glass Factory in Boda, southern Sweden, from September 25th. 2017. We choose to frame the workshop around exploring ways to support processes without fundamentally rethinking the craft of glassblowing. This meant that we focused on how digital fabrication could support glass blowing by developing new tools and forms that could be used in the process of shaping glass at the end of the pipes.

Throughout the one week workshop various artists explored many different avenues of mold making, experienced glassblowers teaming up and working with experienced digital practitioners. Multiple participants already possessed some cross-disciplinary skills and knowledge. The agenda circled around expanding the possibility space of the combination of hot glass and experimental CNC cut forms.

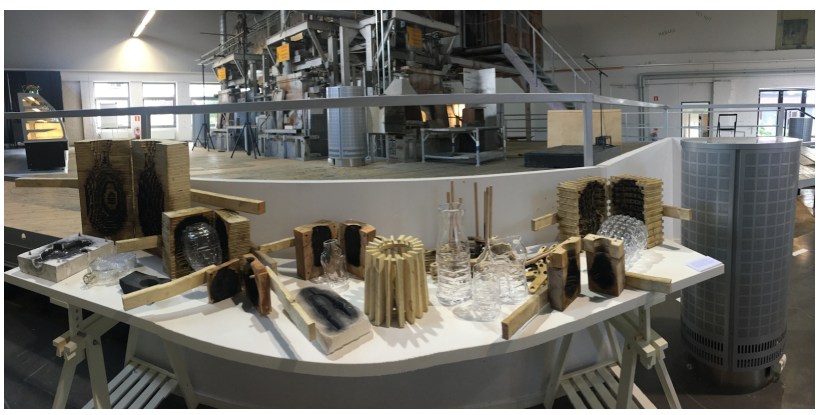

Figure 3. Display of a variety of experimental static and dynamic digitally produced molds.

The workshop had a Maker perspective on two levels. The actual explorations were about the synergies between the digital mold making and the traditional craft. Furthermore, this was done from/with a Maker perspective or method - rapid, explorative iterations of solutions we were not sure whether would work, well knowing that the time cost of cutting another, slightly modified form was low. This was in contrast to the ingrained culture and experience of the craftsmen which was that a mold represented days of handwork and damaging one was a major mishap.

This approach was an antithesis to a classical purposeful design strategy. We did want to get the design right in a Buxton [3] 'sketching' perspective, but there was no utilitarian overarching perspective. The interest was "what will happen we have a bunch of Fablab machines and embed them in a very different context?" How can this tool be used in interesting ways when working with hot glass? It is thus a "what if" perspective on the Maker movement [10] or an exploratory perspective on research through design [14].

The framing of the workshop had the clear intention of a non-hierarchical perspective of exploring the synergies [15]. There were no expectations of one perspective teaching the other the "right way to do it" - instead considering it as two heterogeneous practices with unique knowledge to contribute to each other. The Makers came with a large technical knowledge about digital production and using digital production machinery. The glass blowers came with a large understanding of the intricacies of glass blowing, the material, and the form making traditions. It was important for us to offer these machines "on the glassblowers turf", offering a political agenda of empowering and agency, not obsolescence, replacement or efficiency. In the Fablab movement, we talk about democratisation of access to machinery for people who previously could not Make, but what about access for people who are very good at making, but can presently only Make in a different way?

\section{SEVEN TAKEAWAYS}

Based on the many experiments and discussions during the workshop the following seven generalisable takeaways emerged. We use them as a basis for understanding a potential of the Maker movement, how it can contribute constructively to traditional craft and how traditional craft can enrich the Maker movement.

\section{\#1 Keep distances and iteration cycles short}

Glassblowing is a fast-paced process where you have a relatively short window of opportunity to shape the glass when it comes out of the oven. In many ways this is rapid prototyping on steroids. If something fails in the process it is often easier to throw out the whole piece and start over than it is to correct the problem; this is primarily a consequence of the fact that any flaws remain in the glass and are impossible to remove entirely. Similar processes can be found in any Fablab: if something is cut wrong or the machine creates a flaw half way through, the immediate solution is often to start over and scrap the piece you are working on. With glass, the clock is ticking. 


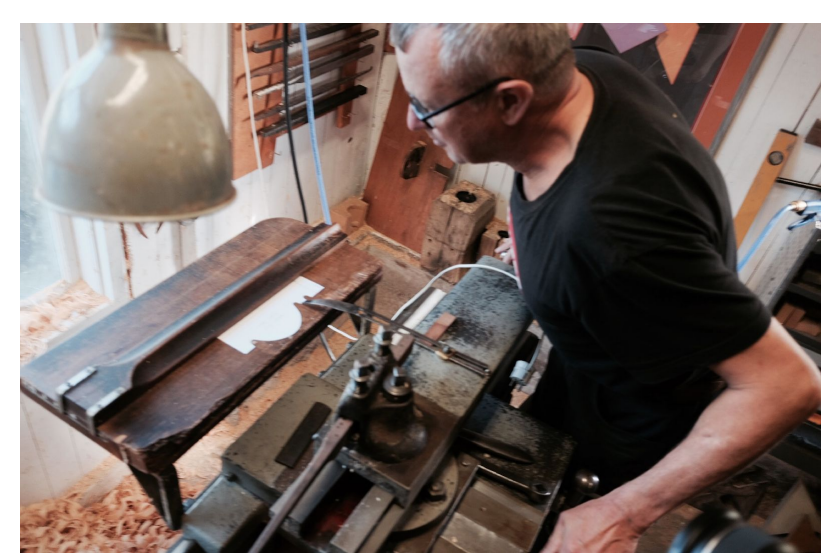

Figure 4. Traditional mold making is done by hand, turning two knobs for the $x$ and $y$ axis respectively.

When it comes to form making however, it was more akin to a slow paced, sacred ritual. The forms were complicated to make and thus great care was taken in designing and preserving them. There was little room for 'dangerous' experimenting, exploring and trying out.

Traditional mold making for glass blowing follows the following steps: The mold-maker makes a 2D outline on paper, in dialogue with the glass blower. This is a process that requires a significant amount of expertise from both parties. The glass blower needs to convey the idea of the mold to the mold maker and together in dialogue they have to adjust the mold so it is realistic to actually blow a shape in it - this requires an integral knowledge of how the hot glass moves inside the mold.

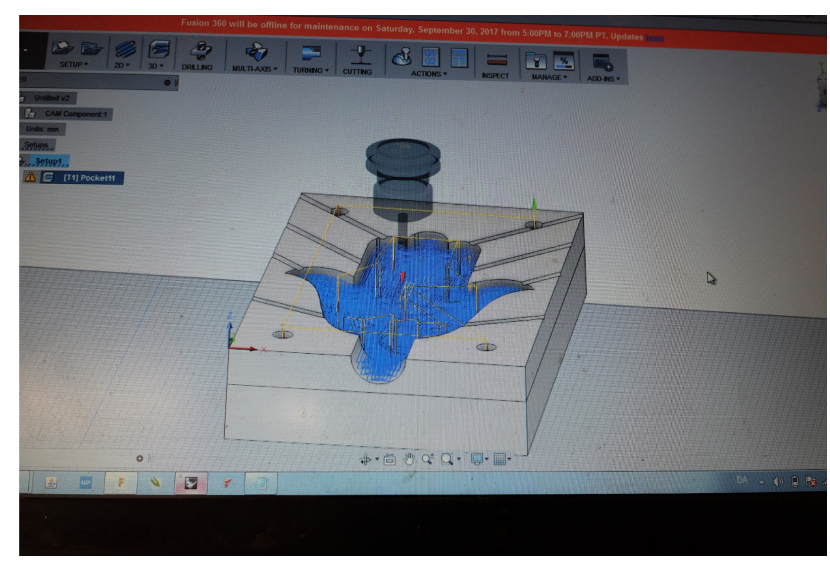

Figure 5. Digital mold making: designing and visualising.

It was clear that digital fabrication has a potential to contribute here. Through digital fabrication we were able to design, modify and redesign quickly, thus creating a rapid prototyping process suitable for what the actual glass blowing process looked like. E.g. a glassblower could try out a form and if it did not match expectations then a new form could be produced by adjusting the digital drawing and a new modified form could be rapidly cut at low (human) cost.

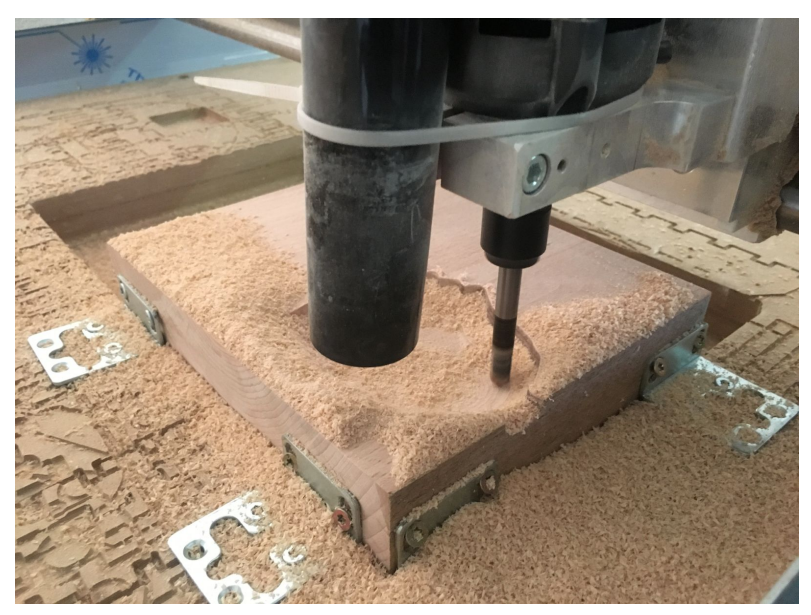

Figure 6. Digital mold making: CNC milling the mold.

Nowadays a CNC machine has a comparable investment cost to a manual lathe, and it can be operated by people who do not have in depth knowledge of manual wood cutting. Furthermore, the $\mathrm{CNC}$ machine was instantly available, located in the same building as the glass blowing workshop - microgeography was important. As simple as this seems, it played a significant role in creating a direct dialogue between form making and glass blowing and added to the potential of creating short iteration cycles through short distance.

\section{\#2 Easy reproduction enables experimentation and exploration}

Introducing the $\mathrm{CNC}$ machine could be seen as a disruption of the traditional mold making process, however this was not our intent or interest during the workshop, for multiple reasons. First and foremost, reproducing molds that could already be created (size, shape and detail wise) on the lathe would have required a time consuming production process that would have been outside the scope of the workshop - and worse, moved the focus from experimenting with what new potentials $\mathrm{CNC}$ based form making offers. Actually working with the machine and material enhanced the learning outcome [11].

One such example is that traditionally molds must be round along the longitudinal axis, as the glassblower needs to turn the glass bubble while blowing into the form. This prevents the glass from burning into the form leaving the wood texture on the glass and damaging the form. With the CNC based production we were less 
concerned about preserving the form because we could more easily cut another one. Thus it became more feasible to explore irregular shapes.

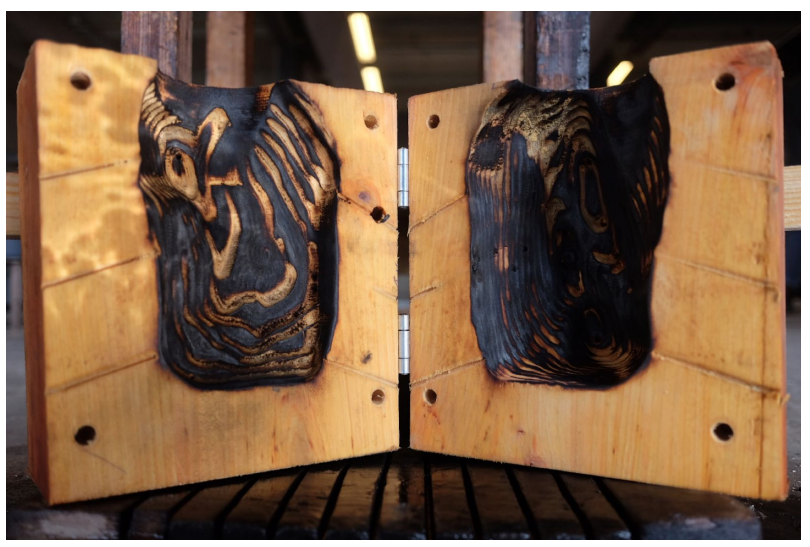

Figure 7. Example of a irregular mold where the glass has charred the surface, because the glass did not rotate as it was blown into the form.

This was done by CNC cutting two halves of a form based on an irregular 3D object. When blowing in the form the glass would become locked into the irregular shape and burn into the wooden form.

From a mass production perspective this was clearly a disadvantage, but from a creative exploratory perspective allowing yourself to burn the mold opened up new possibilities. One iteration of glassblowing would not destroy the form, but burn off the edges of any finer details. Thus it was possible to use the same form many times. Each time less and less details would manifest. From a technical point of view this was unsatisfying, but from an artistic perspective it allowed an interesting tradeoff between reproduction and uniqueness - in a sense each piece became a unique one off piece. When the form became too burned out, it was trivial to reproduce it, or even make new iterations/versions as one went along.

\section{\#3 Limitations as a creative tool}

Similarly to losing detail when the wooden mold was burned, the digital fabrication machines posed multiple limitations which initially could be considered problematic - but could also be seen as inspiration for new expressions. In the invitation to the workshop we wrote "this project is also an invitation to work with the limitations as a part of the creative practice ... we will probably only produce relatively small forms as the $\mathrm{Z}$ axis travel of the machines is only about $10 \mathrm{~cm}$ ". We specifically invited the workshop participants to embrace the limitations of the machinery and digital production method creatively to explore new avenues.

The limitation of a $10 \mathrm{~cm} \mathrm{Z}$-axis is a relatively severe limitation when it comes to producing glassblowing forms because that meant that the outer diameter of the mold could only be $20 \mathrm{~cm}$ wide. During the workshop we started to wonder if we could rethink the fundamental mold production method and use some strategies that are common in the Fablab world when creating 3D shapes from flat components.

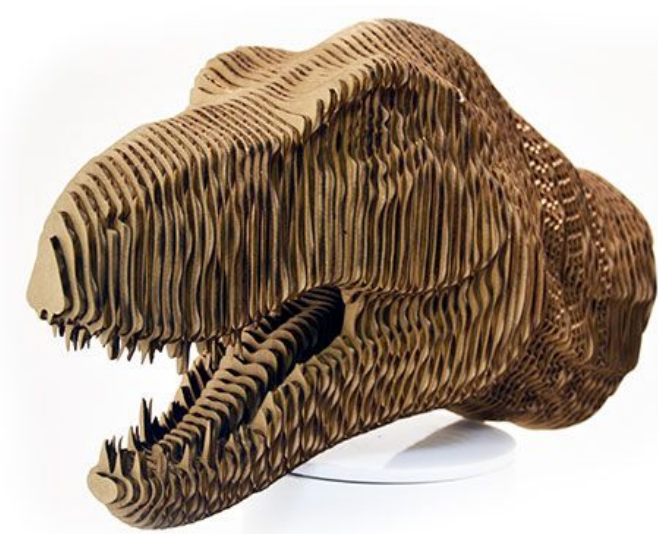

Figure 8. A T-Rex head model that can be downloaded from the Epilog laser website [4].

A common "hello world" object to make on a laser cutter is some version of the T-Rex head. Since a laser cutter can only cut 2D forms, the method is to cut multiple 2D shapes and stack and glue them together to create a 3D form. By using this strategy on negative molds we were able produce forms that exceeded the $10 \mathrm{~cm} \mathrm{Z}$-axis limitation.

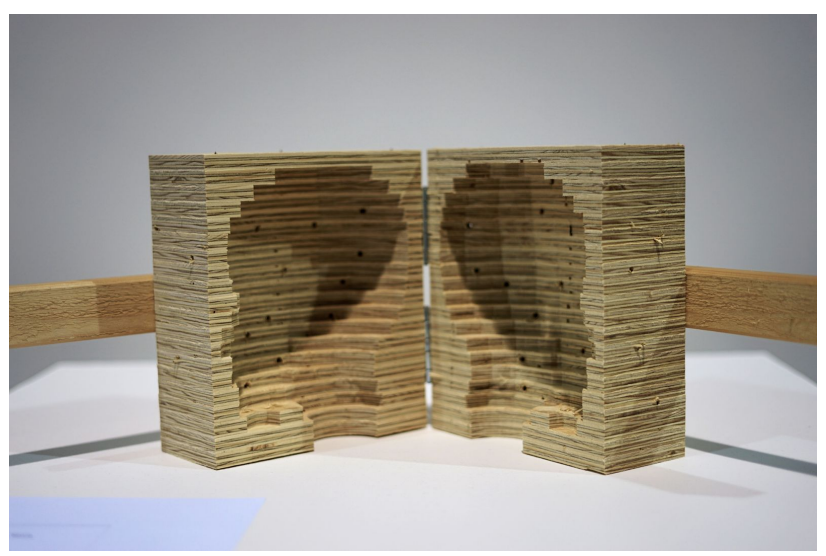

Figure 9. Stacked 2D layers creating a form larger than what could fit within the $Z$-axis. 
Stacking or slicing layers are well known techniques in the Fablab world, but not usually used in the glassblowing world.

As can be seen in figures 9 and 10 the stacking left a "step" like texture to glass blow in. This stepped mold became a potential for wonder - how would the glass form around it? Because of the viscosity of molten glass glassblowing produces rounded shapes and corners. Blowing into this form still produced round shapes, but the aesthetics of the stack were preserved.

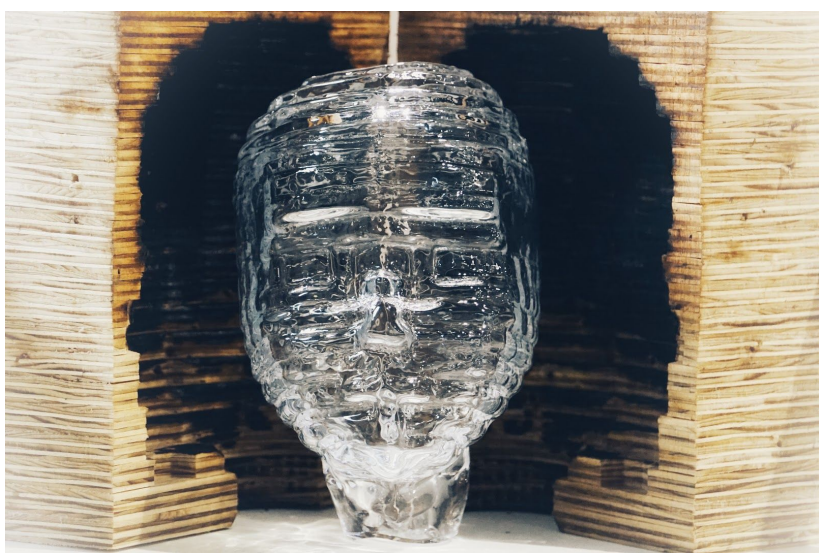

Figure 10. The resulting glass blown object in the stepped stacked CNC cut mold.

This strategy for producing large forms opened up new avenues for exploration in how different slicing and stacking techniques could produce different surface textures. As shown in figures 11 and 12, a grid based structure created large holes in the form that the glass could expand into, resulting in a structure akin to bubble wrap.

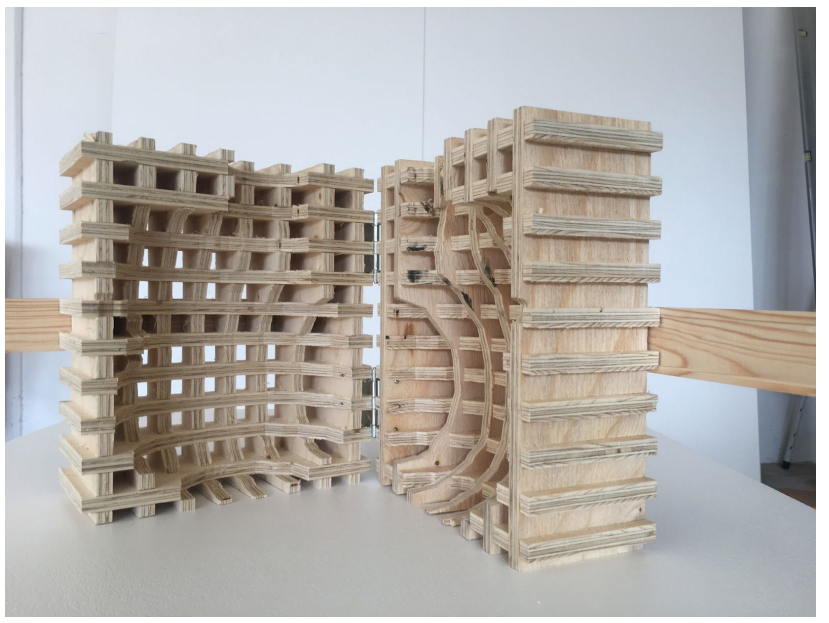

Figure 11. Grid based slicing strategy for a form, which created large holes.

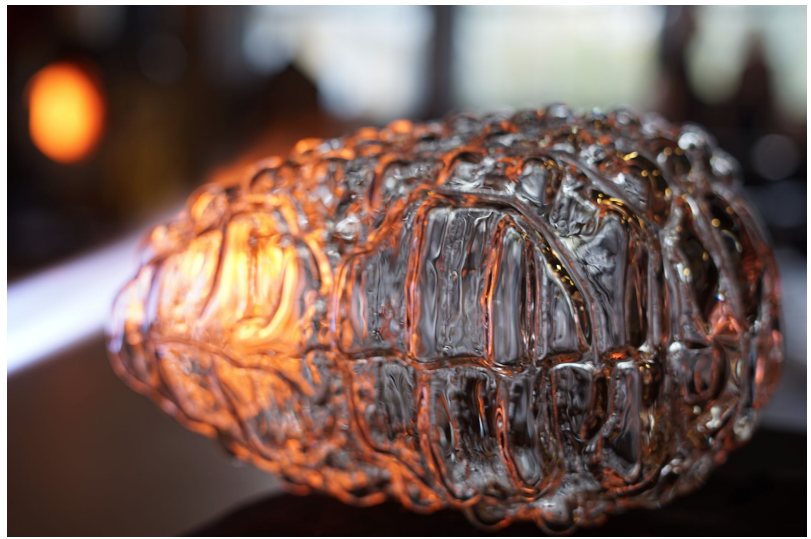

Figure 12. Bubble wrap like surface as consequence of the holes in the form.

Working with the limitations of the production method forced us to explore new avenues. Instead of being in competition to traditional form making it became an exploration into new aesthetic expression.

Fablab Making techniques helped provoke explorative design, non-idiomatic interaction. Related to this, making techniques provided a responsive sketching media. Making was a convenient co-production nexus and yielded artifactual knowledge [21].

\section{\#4 Expanding complexity}

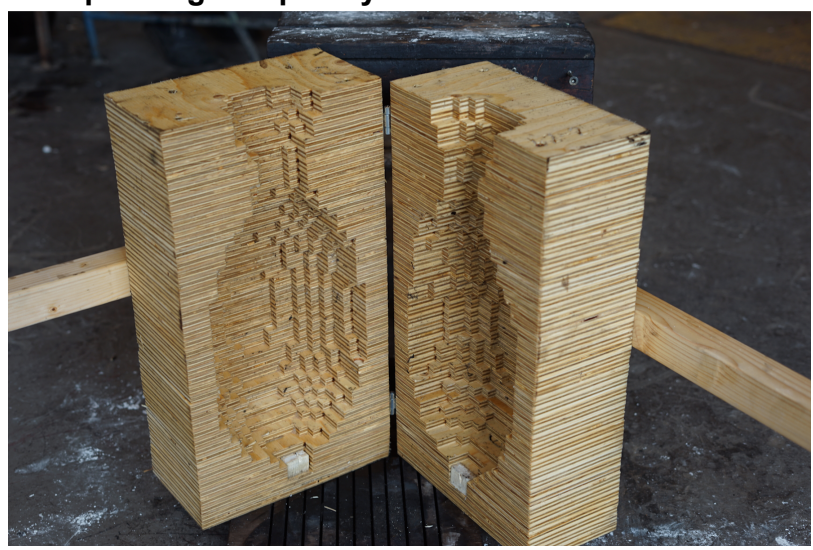

Figure 13. A larger CNC cut stacked mold.

Similar to the stacking method explored in the previous takeaway, we explored more algorithmic strategies for designing form. This was done by pixelating a traditional vase form and creating a stacked negative where the pixelation size matched the stacking (i.e. the horizontal steps were the same size as the vertical steps), thus turning the stacked pattern into a pixelated pattern.

This level of complexity is not impossible by hand, but is significantly more feasible with computer controlled machines. 
Outsourcing complex details to a machine enables the artist to use more focus and time on other aspects.

This aligns well with the praxis of the maker movement, in which it is possible to do things that otherwise would require a significant amount of manual labour.

We expect to see a "renaissance" in both art, design, architecture etc., as robotic machines transform the price of detail, patterns and complexity, from prohibitive to almost free. We have previously worked with this avenue [13] when we designed and built a large scale 3D concrete printer.

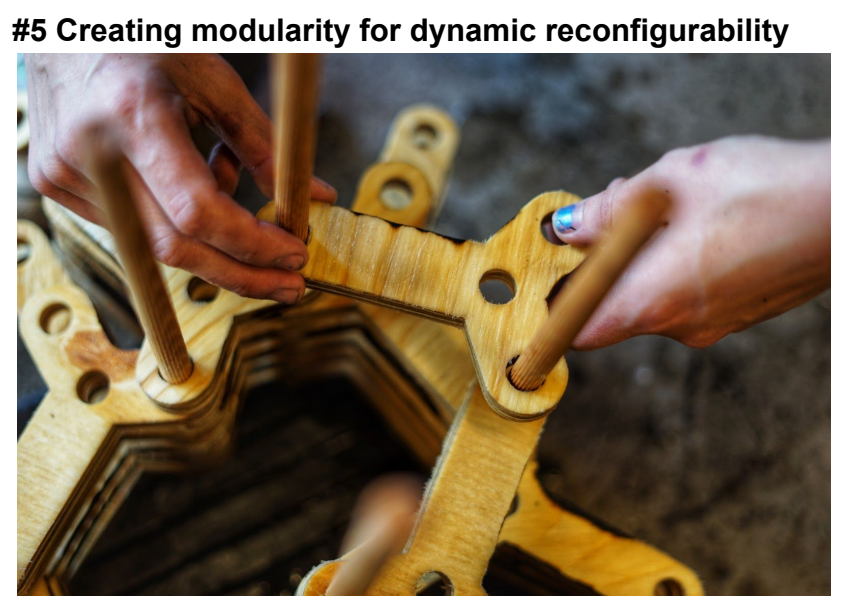

Figure 14. Glass artist experimenting with modular shapes to create unique and reconfigurable molds.

Digital fabrication machines never become tired of repetition. Creating the same shape over and over again is a matter of copy paste. Furthermore, it is possible to quickly make different versions of the same shape by adjusting the shapes before they are produced in larger quantities.

This quality was explored during the workshop. A set of shapes were created and produced in large batches. The shapes were designed with holes so they could be combined in endless ways, connected together with wooden dowels. The mold was thus created by combining these shapes in various ways. The final form was thus a consequence of how the shapes were configured.

The mechanically hinged joints gave the possibility for live, realtime shaping of the glass as it was blown into the form. The assistant holding the form for the glassblower became a codesigner in the process by manipulating the form of the mold. The form became dynamic in the actual glass blowing process. Using digital fabrication and modular pieces, it would be possible to create flexible kits for glassblowers. They would be able to create their own lego like pieces in a Fablab facility and use them as a part of their tool repertoire in the glass studio.

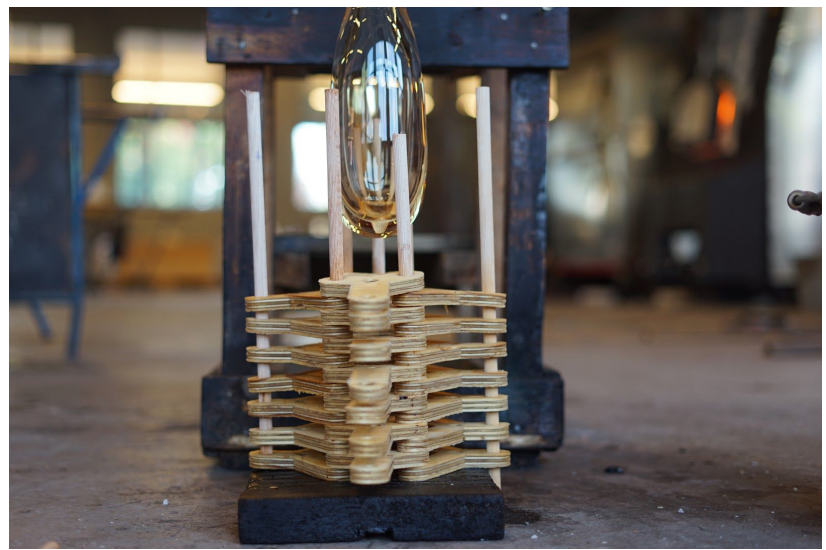

Figure 15. One possible combination of the modular pieces.

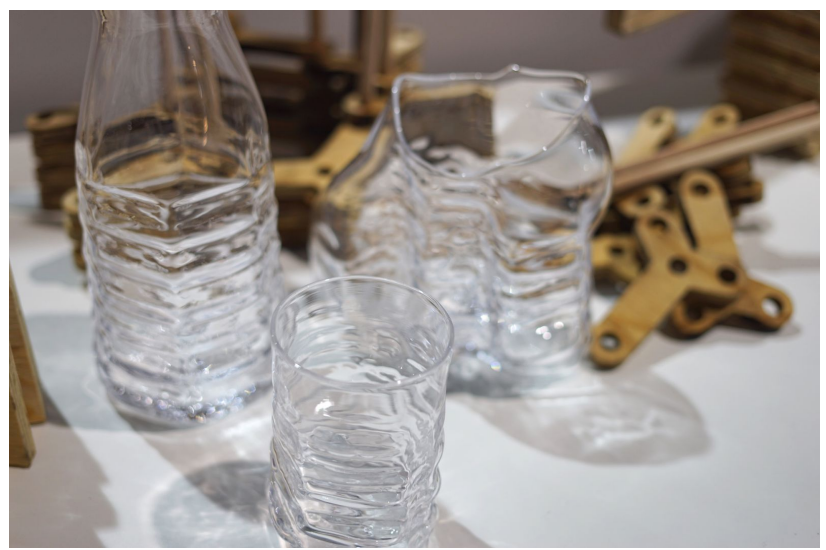

Figure 16. Examples of different molds that were created through the configurable shapes.

\#6 Craftsman knowledge can save the maker movement from uniform aesthetics and material limitations

Artefacts created in the Fablab/Maker movement are often easily recognisable. Tools are often $3 \mathrm{~d}$ printer, laser cutter, CNC mill and Arduino. Materials are often acrylic, plywood or HDF. Form is often finger boxes, rectangular shapes.

Utilizing the material knowledge endemic in "trades", from glassblowing to pottery to weaving, can enable the Maker movement to widen its horizons, use more materials, and solve more challenges. With help from "crafts" domains, suddenly the maker movement can help create things that could last a hundred years, or withstand 10,000 volts, or be waterproof or acid resistant. 2D making (laser or $\mathrm{CNC}$ cutting flat sheets) can be 
transformed to 3D making in organic forms, using the medium of glass.

\section{\#7 Glimse of a possible co-learning model: reciprocal apprenticeship \\ We would like to explore using the term reciprocal apprenticeship to describe the equal, bilateral collaborative learning form we set up and witnessed during the workshop.}

Building on apprenticeship and situated learning [20], there are concepts like collaborative apprenticeship [9], featuring reciprocal interactions, but these usually seem to be either framed in terms of between colleagues where one is clearly the master and the other the apprentice (i.e. a technically competent teacher mentoring another teacher) - or betweens peers learning at the same or similar levels such as pupils in a school environment. In our emerging understanding, reciprocal apprenticeship could specifically focus on two highly skilled craftsmen from two different fields teaching each other simultaneously. We should however clarify that this is an inspiration gained from the workshop more than a description - in fact multiple participants were cross-disciplinary and already had skills spanning both fields.

By framing learning in Maker movement terms of collaboratively, playfully setting a goal - and the glassblower being the master in one field and simultaneously the apprentice int the other field, the CNC expert being the master in one field and at the same time the apprentice in the other, learning and exploring innovative paths toward the shared goal becomes a joint effort and it becomes unclear who the teacher is and who the student is, and unclear which field is giving the other field the greater benefit. This approach warrants further exploration with heterogeneous settings and participants.

\section{DISCUSSION}

It is relevant to reflect on the role of the Maker movement and the meeting between craft and Maker in the described case and wider, in a more generalisable fashion.

\section{From disruption to synergies}

Discussion of traditional crafts and new digital technologies, machines and robots all too often end in a binary dichotomy, of replacement, obsolescence, survival. Likewise, computer scientists and traditional craftspeople are all to often in different silos, not inspiring, enriching and working alongside each other.
By attempting to frame the meeting as synergy, voluntary possibilities, not "disruption", an inspiring artistic meeting could take place, where the digital is viewed as a new tool, a new possibility; not a threat. By working together and acknowledging each other's areas of expertise, often knowledge gleaned over decades or centuries, it can become clear what computer scientists and traditional craftspeople have to offer each other.

Within technological industries disruption is a buzzword a hunt for disruption and the next business opportunity. In many ways glass blowing has already been disrupted by industrial form-based glass production. Thus, as tantalizing it might be to frame the efforts of exploring digital production within studio glass blowing as a disruptive strategy, it would be a strained concept.

\section{Limits of the maker movement}

Products produced in Fablabs sometimes suffer a lack of diversity in production method, aesthetic and materials. Often a laser cut finger box, made of acrylic or plywood is produced with Fablab machinery. Interacting with crafts can widen the horizons of both groups - Making can be far broader than the most common Fablab machinery: $3 \mathrm{~d}$ printer, $\mathrm{CNC}$ machine and laser cutter. On the other hand, making the digital physical; precision and repeatability with modifications - offer options traditional crafts have lacked.

As Hobye [13] points out, the Maker movement falls under the $80 / 20$ rule of being good at exploring potentials, but less good at creating finished products. A similar observation can be found here. The workshop became a generative understanding of the potential of combining the two fields, more than fundamentally rethinking either of the two.

\section{Expanding our perception of what the maker movement is}

The advantage for the Maker movement in including craftspeople is the possibility for wide-ranging extension of the materials and processes possible in a Fablab or Makerspace, acknowledging Making as a broader field than the currently common suite of Fablab machinery.

We postulate Fabmaking could benefit from being expanded with a wider selection of materials than plexiglass, wood and $3 \mathrm{~d}$ printed plastic. This is far easier with the domain-specific expertise of traditional craftspeople, gleaned and refined through centuries. Instead of trying to make everything out of laser cut plexiglass and plywood, new possibilities arise, using a combination of craft and maker machines. This provides 
both creative and practical advantages. Artistic, aesthetic and material possibilities are multiplied. Products and applications which would be difficult or impossible to achieve using only Fablab machines become possible, even easy. New hybrid or multi-stage processes can be developed. For example, it is not easy to make a drinking glass, or anything watertight and easily cleanable, on a laser cutter. It is not easy to make something with a high heat tolerance in a standard 3D printer. Glass as a second stage Maker material makes multiple problems solvable: glass is fireproof, heat tolerant, chemical resistant, cleanable, very long lasting, and electrically insulating up to very high voltages, in a way which many materials which are normally easily processable in a Fablab are not.

\section{Cultural meeting}

The workshop was a meeting of cultures - which went well. Perhaps because the glassblowers felt agency, not threat? Because the $\mathrm{CNC}$ machines moved to the glassblowery, not vice versa?

By acknowledging and recognising the great knowledge, expertise, and experience of traditional craftsmen, leaving them in charge of the creative process, and offering digital machines as a possibility to explore, an extension or modification of a known and familiar production step, it was possible to frame new tools as an option, a new possibility, not a threat supplanting the whole process with a robot.

\section{CONCLUSION \& PERSPECTIVES}

This paper only highlights some potential of synergies and points to near future potential. For the field to evolve we need to conduct more workshops, more research, into more craft fields, and experiment with more cultural meetings, working together longer term. Further work includes examining the dynamics: Fablabbing could challenge industrial processes. DIY digital supported design/craft can challenge the design professionals, while bringing new creative friction to the table. Digital Bauhaus [28] is an example of craft ambitions seen through the lens of Making. More rapid DIY tools mean that amateurs can make more 'professional' products, with more finish - not just first stage functioning prototypes.

\section{ACKNOWLEDGEMENTS}

The workshop was a part of NF funded project, 'Scandinavian Glass - starting all over'. Organized by The Glass Factory / Maja Heuer. The workshop was done as a collaboration between Fablab RUC and The Glass Factory.

Participants: Noam Dover, Jason Knight, Helena Kågebrand, Mads Hobye, Ammy Olofsson, Michaela Nina Wallachova, Bjørn Friborg, Mikael Jacobsson.

Specific accreditation on pieces: Mads Hobye: Glass blown heads. Jason Knight: Expanded complexity vase. Ammy Olofsson: Dynamic forms. Noam Dover: Irregular shapes

\section{REFERENCES}

1. Chris Anderson. 2014. Makers: the new industrial revolution. Crown Business.

2. John Bowers. 2012. The logic of annotated portfolios: communicating the value of 'research through design'. In Proceedings of the designing interactive systems conference, DIS '12 (pp. 68-77). New York, NY, USA: ACM. http://doi.acm.org/10.1145/2317956.2317968

3. Bill Buxton. 2008. Sketching User Experiences. Getting the design right and the right design, Focal Press.

4. Epilog 2018. https:/www.epiloglaser.com/resources/sample-cl $\underline{\mathrm{ub} / \text { trex-head-3d-model.htm (retrieved }}$ 2018-03-18)

5. Fablabs.io, Fab Foundation. 2018. List of Fablabs worldwide. https://www.fablabs.io/labs (retrieved 2018-03-18)

6. William Gaver. 2012. What should we expect from research through design?, CHI '12 Proceedings of the SIGCHI Conference on Human Factors in Computing Systems. 937-946. https://doi.org/10.1145/2207676.2208538

7. Bill Gaver, John Bowers. 2012. Annotated portfolios. interactions, 19(4), 40-49. New York, NY, USA: ACM. http://doi.acm.org/10.1145/2212877.2212889

8. Graham Gibbs. 1999. Using assessment strategically to change the way students learn, in Brown, S. and Glasner, A. (eds.) Assessment Matters in Higher Education: Choosing and Using Diverse Approaches, Maidenhead: SRHE/Open University Press.

9. Evan Glazer, Michael J. Hannafin, Liyan Song. 2005. Promoting technology integration through 
collaborative apprenticeship. Educational Technology Research and Development. December 2005, Volume 53, Issue 4, pp 57-67.

10. Michael Haldrup, Mads Hobye, Kristine Samson, Nicolas Padfield. 2015. Remix Utopia. Eleven Propositions on Design and Social Fantasy. In Nordes No 6. Stockholm: Nordic Design Research Conference.

11. Michael Haldrup, Mads Hobye, Nicolas Padfield. 2016 The Bizarre Bazaar: FabLabs as Hybrid Hubs. Critical Edge Conference. Roskilde.

12. Mark Hatch. 2013. The Maker Movement Manifesto: Rules for Innovation in the New World of Crafters, Hackers and Tinkerers. McGraw-Hill.

13. Mads Hobye, Nicolas Padfield, Michael Haldrup. 2016. Making makers make maker machines, Fab12. Shenzhen.

14. Mads Hobye, Jonas Löwgren. 2011. Touching a stranger: Designing for engaging experience in embodied interaction. Int. J. Design 5, 3, 31--48.

15. Tim Ingold. 2010. Bringing Things Back to Life: Creative Entanglements in a World of Materials. NCRM.

16. Tim Ingold. 2013. Making: Anthropology, Archaeology, Art and Architecture. Routledge.

17. Timothy Ingold, Caroline Gatt. 2013. From Description to Correspondence: Anthropology in Real Time. Design Anthropology - Theory and Practice. Bloomsbury.

18. Thomas Kalil. 2013. Have Fun-Learn Something, Do Something, Make Something, in Design, Make, Play - Growing the Next Generation of STEM Innovators

19. Ilpo Koskinen, John Zimmerman, Thomas Binder, Johan Redstrom, Stephan Wensveen. 2011. Design Research Through Practice - From the Lab, Field, and Showroom. Morgan Kaufmann
20. Jean Lave, Etienne Wenger. 1991. Situated learning: Legitimate peripheral participation. Cambridge university press.

21. Jonas Löwgren. 2016. On the significance of making in interaction design research. Interactions xxiii(3):26-33.

22. Lee Martin. 2015. The Promise of the Maker Movement for Education. Journal of Pre-College Engineering Education Research (J-PEER), Volume 5, Issue 1, Article 4 https://doi.org/10.7771/2157-9288.1099

23. Erin O'Connor. 2005. Embodied knowledge: The experience of meaning and the struggle towards proficiency in glassblowing. http://journals.sagepub.com/doi/abs/10.1177/146 6138105057551

24. Nicolas Padfield, Michael Haldrup, Mads Hobye. 2014. Empowering academia through modern fabrication practices. Fablearn EU 2014. https://forskning.ruc.dk/da/publications/empower ing-academia-through-modern-fabrication-practi $\underline{\text { ces }}$

25. Barak Rosenshine, Carla Meister. 1992. The Use of Scaffolds for Teaching Higher-Level Cognitive Strategies. Educational Leadership, v49 n7 p26-33 Apr 1992

26. David Wood, Jerome S. Bruner, Gail Ross 1976. The role of tutoring in problem solving. Journal of Child Psychology and Child Psychiatry, 17, 89-100.

27. John Zimmerman, Jodi Forlizzi, Shelley Evenson. 2007. Research through design as a method for interaction design research in HCI. In Proceedings of the SIGC-HI conference on human factors in computing systems, CHI '07 (pp. 493-502). New York, NY, USA: ACM. http://doi.acm.org/10.1145/1240624.1240704

28. Pelle Ehn. 1998. Manifesto for a digital bauhaus. Digital Creativity. Vol 9(4) p. 207-217. Taylor \& Francis. 